\title{
Some New Classes of Consistent Risk Measures
}

\author{
Marc J. Goovaerts ${ }^{a, b}, \quad$ Rob Kaas $^{b}, \quad$ Jan Dhaene ${ }^{a, b}$, Qihe Tang ${ }^{b}$ \\ ${ }^{a}$ CRIS, Catholic University of Leuven \\ ${ }^{b}$ Department of Quantitative Economics, University of Amsterdam
}

May 9, 2003

\begin{abstract}
Many types of insurance premium principles and/or risk measures can be characterized by means of a set of axioms which in many cases are arbitrarily chosen and not always in accordance with economic reality. In the present paper we generalize Yaari's risk measure based on some less stringent axioms. In addition we derive translation invariant minimal Orlicz risk measures, which we call Haezendonck risk measures, and obtain sufficient conditions on the risk measure of Bernoulli risks to fulfill additivity and superadditivity properties for Orlicz premium principles.
\end{abstract}

Keywords: Consistent risk measures, Haezendonck risk measure, Yaari's dual theory of choice under risks

\section{Introduction}

Recently, in Goovaerts et al. $\left(2003_{a}\right)$ it was argued that risk measures should be selected in an appropriate way in order to reflect the basic economic underlying reality. Indeed several examples can be given, which are relevant to real life insurance problems where evidently the properties that the risk measures should have are determined by the realities of the actuarial applications.

Example 1.1 (Insurance - Reinsurance). Suppose that a risk $X$ is split into two parts as

$$
X=\left[X-(X-d)_{+}\right]+(X-d)_{+}
$$

Clearly, both parts are comonotonic because they are increasing functions in $X$, and thus a principle, say $\pi[\cdot]$, that is additive for comonotonic risks such that

$$
\pi[X]=\pi\left[X-(X-d)_{+}\right]+\pi\left[(X-d)_{+}\right]
$$

has some advantages for distributing the premium between the two parts involved. It provides e.g. a tool to compare the part of the premiums charged for the risk $(X-d)_{+}$with 
the reinsurance premiums $\pi_{R}\left[(X-d)_{+}\right]$that is actually charged by the reinsurer. The same also holds for the case

$$
X=a X+(1-a) X, \quad 0 \leq a \leq 1
$$

which leads to

$$
\pi[X]=\pi[a X]+\pi[(1-a) X]
$$

since the principle $\pi[\cdot]$ is additive for comonotonic risks. In both situations comonotonic additive risk measures are consistent with the practical problems at hand. Related discussions can be found e.g. in Wang (1996).

Example 1.2 (Premium Calculation). For practical reasons, in the case of premium calculation a splitting of the risk into two parts $a X$ and $(1-a) X$ for some $0<a<1$ should not lead to a decrease in premiums. Hence

$$
\pi[X] \leq \pi[a X]+\pi[(1-a) X]
$$

In this case subadditive risk measures for comonotonic risks are consistent with this particular situation. This property has been called the subdecomposability of a risk in Goovaerts et al. (1984).

Example 1.3 (Premium Calculation from Top-down). As in Bühlmann (1970) (see also Gerber $(1979,1985)$ and Kaas et al. (2001)), suppose that one approaches a premium calculation from top-down, e.g. by considering a ruin probability model for the determination of the portfolio premium on the top level. In case of e.g. motorcar insurance the risks constituting the portfolio can generally be considered to be independent. Then, in order to distribute the premium income at the top level among the risks at the down level, use of an additive risk measure for independent risks,

$$
\pi\left[X_{1}^{\perp}+X_{2}^{\perp}\right]=\pi\left[X_{1}^{\perp}\right]+\pi\left[X_{2}^{\perp}\right]
$$

is consistent with the situation at hand.

Example 1.4 (Capital Allocation). In order to keep the residual risk after the capital has been allocated of the conglomerate under control in the sense that the risk of the conglomerate benefits from the diversification, a superadditive risk measure is consistent with this situation because

$$
\pi\left[X_{1}+X_{2}\right] \geq \pi\left[X_{1}\right]+\pi\left[X_{2}\right]
$$

results in the residual capital to be asked to the shareholders satisfying

$$
\left(X_{1}+X_{2}-\pi\left[X_{1}+X_{2}\right]\right)_{+} \leq_{1}\left(X_{1}-\pi\left[X_{1}\right]\right)_{+}+\left(X_{2}-\pi\left[X_{2}\right]\right)_{+} \cdot
$$


Example 1.5 (Solvency Margin). Consider the Bernoulli $(q)$ risk $B_{q}, q \in[0,1]$. Then for any $a>0$ the risk measure $\pi\left[a B_{q}\right]$ should be increasing in $q \in[0,1]$ if this risk measure $\pi[\cdot]$ is used as a premium rule. However, when one aims at calculating a "solvency margin" (i.e. a provision for the adverse outcome, or a claim that is larger than the expected one) $\pi\left[a B_{q}\right]$ for this Bernoulli risk, it is clear that $\pi\left[a B_{0}\right]=\pi\left[a B_{1}\right]=0$ because in both situations there is no uncertainty involved. One could think about $\pi\left[a B_{q}\right]=\pi\left[a B_{1-q}\right]$ to express the equality of uncertainty between the two risky situations $a B_{q}$ and $a B_{1-q}$. One could also consider the financial picture in the following sense by means of an actuarial safety loading $\lambda>0$ :

$$
\mathrm{E}\left[B_{q}\right](1+\lambda)=q(1+\alpha(1-q))=q+\alpha q(1-q)
$$

where $\alpha$ is a proportional part of the excess amount $1-q$ above the expected claim size $q$. For a risk with $q^{\prime}=1-q$, one gets $(1-q)(1+\alpha q)=(1-q)+\alpha(1-q) q$. Consequently, $\pi\left[B_{q}\right]=\pi\left[B_{1-q}\right]=\alpha q(1-q)$ is an example of a consistent risk measure for calculating solvency margins.

The previous examples indicate that each realistic situation needs a specific set $\mathbb{S}$ of axioms. We introduce the following definition:

Definition 1.1. Let $\mathbb{S}$ be a set of axioms for risk measures, and $\alpha, 0<\alpha<1$, be a level. A risk measure $\pi[\cdot]=\pi_{(\mathbb{S}, \alpha)}[\cdot]=\pi_{\alpha}[\cdot]$ is called $(\mathbb{S}, \alpha)$-consistent if $\pi[\cdot]$ is a rule that assigns a value to each risk $X$ satisfying the axioms $\mathbb{S}$ and such that $\pi[X] \geq F_{X}^{-1}(\alpha)$, where $F_{X}^{-1}(\alpha)$ is the $\alpha$ th quantile of the risk $X$, and is defined, as usual, by $F_{X}^{-1}(\alpha)=\inf \{x: F(x) \geq \alpha\}$.

In the present paper we first generalize some of the consistent risk measures for the following choice $\mathbb{S}_{1}$ of axioms:

A1. $X \leq_{1} Y \Longrightarrow \pi[X] \leq \pi[Y]$, where $\leq_{1}$ denotes "stochastically not greater than";

A2. $\pi\left[X^{c}+Y^{c}\right]=\pi\left[X^{* c}+Y^{c}\right]$ provided that $\pi[X]=\pi\left[X^{*}\right]$

A3. $\pi\left[X_{n}\right]$ converges to $\pi[X]$ if $F_{X_{n}}$ is non-decreasing and converges weakly to $F_{X}$.

Clearly, $\mathbb{S}_{1}$ is less restrictive than the axioms of Yaari's (1987) dual theory of choice under risks because, according to Yaari's axioms,

$$
\pi\left[X^{c}+Y^{c}\right]=\pi\left[X^{c}\right]+\pi\left[Y^{c}\right]=\pi\left[X^{* c}+Y^{c}\right]
$$

must hold and hence A2 follows immediately. We notice that the first axiom is not always valid in case one uses a risk measure as a solvency measure as was explained in Example 1.5.

Then, we consider another choice $\mathbb{S}_{2}$ of axioms as follows: 
B1. $X \leq_{1} Y \Longrightarrow \pi[X] \leq \pi[Y]$

B2. $\pi[a X]=a \pi[X]$ for $a>0$;

B3. $\pi\left[X_{1}+X_{2}\right] \leq \pi\left[X_{1}\right]+\pi\left[X_{2}\right]$

This is the set of axioms satisfied by an Orlicz premium principle; see Goovaerts et al. (1984, $\left.2003_{b}\right)$. By considering Orlicz distances for the risk $(X-u)_{+}$and determining $u$ optimally one obtains in addition a translation invariance property for this risk measure; see Section 3 , where we will introduce the Haezendonck risk measure.

\section{Generalized Yaari risk measure}

For simplicity, in this section when we mention a risk variable we mean that it is a nonnegative random variable. The result below can easily be extended to the more general case where the risk variable takes values from the whole real line.

Let $\pi[\cdot]$ be a risk measure, which is such that, for a risk variable $X$, the value $\pi[X]$ is uniquely determined by its distribution function $F_{X}$. We write $X \sim Y$ if $\pi[X]=\pi[Y]$ and denote by $B_{q}$ with $0 \leq q \leq 1$ a Bernoulli variable which satisfies

$$
B_{q}= \begin{cases}1 & \text { with probability } q, \\ 0 & \text { with probability } 1-q .\end{cases}
$$

In terms of the risk measure $\pi[\cdot]$ and the Bernoulli variable $B_{q}$, we introduce two functions $f(\cdot)$ and $v(\cdot, \cdot)$ by

$$
f(x)=\pi[x], \quad v(x, q)=\pi\left[x B_{q}\right],
$$

where $x \geq 0$ and $0 \leq q \leq 1$. Clearly, $f(x)=v(x, 1)$ for each $x \geq 0$.

Theorem 2.1. Let the risk measure $\pi[\cdot]$, the functions $f(\cdot)$ and $v(\cdot, \cdot)$ be given as above such that

$$
w(q)=\left.\frac{\partial}{\partial x} v(x, q)\right|_{x=0}
$$

exists and is non-decreasing in $q \in[0,1]$ with $w(0)=0$ and $w(1)=f^{\prime}(0)>0$. Then $\pi[\cdot]$ satisfies the following properties:

A1. $X \leq_{1} Y \Longrightarrow \pi[X] \leq \pi[Y]$

A2. $\pi\left[X^{c}+Y^{c}\right]=\pi\left[X^{* c}+Y^{c}\right]$ provided that $\pi[X]=\pi\left[X^{*}\right]$

A3. $\pi\left[X_{n}\right]$ converges to $\pi[X]$ if $F_{X_{n}}$ is non-decreasing and converges weakly to $F_{X}$ 
if and only if the function $f(\cdot)$ is continuous and non-decreasing on $[0,+\infty)$ and

$$
\pi[X]=f\left(\frac{1}{w(1)} \int_{0}^{+\infty} w\left(1-F_{X}(x)\right) \mathrm{d} x\right) .
$$

\section{Proof. 1. First we prove the "if" part.}

Assume that $(2.3)$ holds with the function $f(\cdot)$ continuous and non-decreasing on $[0,+\infty)$. The proofs for A1 and A3 are trivial. So we only need to prove A2. For any risk variable $X$, in case $\int_{0}^{+\infty} w\left(1-F_{X}(x)\right) \mathrm{d} x=+\infty$, by (2.3) it holds that $\pi[X]=\pi\left[X^{*}\right]=f(+\infty)$. Hence by property A1 we can conclude that $\pi\left[X^{c}+Y^{c}\right]=\pi\left[X^{* c}+Y^{c}\right]=f(+\infty)$, which indicates A2. Symmetrically, the same discussion can be given for the case where $\int_{0}^{+\infty} w\left(1-F_{X^{*}}(x)\right) \mathrm{d} x=$ $+\infty$. Thus it remains to prove A2 for the case where

$$
\int_{0}^{+\infty} w\left(1-F_{X}(x)\right) \mathrm{d} x<+\infty \quad \text { and } \quad \int_{0}^{+\infty} w\left(1-F_{X^{*}}(x)\right) \mathrm{d} x<+\infty .
$$

We apply an approximation device to prove the result. Write $Y_{n}=\min \{Y, n\}, n=1,2, \cdots$. The first relation in (2.4) indicates that

$$
\begin{aligned}
\int_{0}^{+\infty} w\left(1-F_{X^{c}+Y_{n}^{c}}(x)\right) \mathrm{d} x & \leq \int_{0}^{+\infty} w\left(1-F_{X}(x-n)\right) \mathrm{d} x \\
& =n w(1)+\int_{0}^{+\infty} w\left(1-F_{X}(x)\right) \mathrm{d} x \\
& <+\infty .
\end{aligned}
$$

Thus, for each $n=1,2, \cdots$, applying integration by parts we have

$$
\begin{aligned}
\pi\left[X^{c}+Y_{n}^{c}\right] & =f\left(\frac{1}{w(1)} \int_{0}^{1} F_{X^{c}+Y_{n}^{c}}^{-1}(1-y) w(\mathrm{~d} y)\right) \\
& =f\left(\frac{1}{w(1)} \int_{0}^{1}\left(F_{X}^{-1}(1-y)+F_{Y_{n}}^{-1}(1-y)\right) w(\mathrm{~d} y)\right) .
\end{aligned}
$$

Define the inverse function of $f(\cdot)$, as usual, by $f^{-1}(y)=\inf \{x: f(x) \geq y\}$. It follows immediately from (2.3) that

$$
\pi\left[X^{c}+Y_{n}^{c}\right]=f\left(f^{-1}(\pi[X])+\frac{1}{w(1)} \int_{0}^{1} F_{Y_{n}}^{-1}(1-y) w(\mathrm{~d} y)\right) .
$$

Symmetrically, it holds that

$$
\pi\left[X^{* c}+Y_{n}^{c}\right]=f\left(f^{-1}\left(\pi\left[X^{*}\right]\right)+\frac{1}{w(1)} \int_{0}^{1} F_{Y_{n}}^{-1}(1-y) w(\mathrm{~d} y)\right) .
$$

This proves

$$
\pi\left[X^{c}+Y_{n}^{c}\right]=\pi\left[X^{* c}+Y_{n}^{c}\right]
$$


for each $n=1,2, \cdots$. Finally, by property A3, letting $n \rightarrow+\infty$ on both sides of (2.5) yields that $\pi\left[X^{c}+Y^{c}\right]=\pi\left[X^{* c}+Y^{c}\right]$.

\section{Next we prove the "only if" part.}

Clearly, from properties A1 and A3, the function $f(x)$ is non-decreasing and continuous in $x \in[0,+\infty)$, the bivariate function $v(x, q)$ is also non-decreasing and continuous both in $x \in[0,+\infty)$ and in $q \in[0,1]$. It follows immediately from (2.2) that

$$
x B_{q} \sim f^{-1}(v(x, q)) .
$$

Now we formulate the remaining proof into two steps.

2.1. At the first step we only consider a special case where the risk variable $X$ has a discrete distribution function with finite supporting points $x_{0}<x_{1}<\cdots<x_{n}$, satisfying

$$
\left\{\begin{array}{l}
\operatorname{Pr}\left[X=x_{i}\right]=p_{i} \geq 0, \quad i=0,1, \cdots, n, \\
\sum_{i=0}^{n} p_{i}=1
\end{array}\right.
$$

Without loss of generality we assume $x_{0}=0$. We define

$$
\Delta_{i}=\left\{\begin{array}{ll}
x_{i}-x_{i-1} & \text { with probability } p_{i}+\cdots+p_{n}, \\
0 & \text { with probability } p_{0}+\cdots+p_{i-1},
\end{array} \quad i=1, \cdots, n .\right.
$$

So $\Delta_{i}$ satisfies

$$
\Delta_{i}={ }^{d}\left(x_{i}-x_{i-1}\right) B_{p_{i}+\cdots+p_{n}}, \quad i=1, \cdots, n,
$$

where ${ }^{d}$ denotes "of the same distribution as". It is not difficult to see that

$$
X={ }^{d} F_{X}^{-1}(U)=\sum_{i=1}^{n} F_{\Delta_{i}}^{-1}(U)={ }^{d} \sum_{i=1}^{n}\left(x_{i}-x_{i-1}\right) B_{p_{i}+\cdots+p_{n}}^{c},
$$

where and throughout, for a random vector $\left(Y_{1}, \cdots, Y_{n}\right)$, we write by $\left(Y_{1}^{c}, \cdots, Y_{n}^{c}\right)$ a random vector with marginals the same as that of $\left(Y_{1}, \cdots, Y_{n}\right)$. Hence

$$
\pi[X]=\pi\left[\sum_{i=1}^{n}\left(x_{i}-x_{i-1}\right) B_{p_{i}+\cdots+p_{n}}^{c}\right] .
$$

Recall (2.6) and property A2. For any integer $m \geq 1$ we have that

$$
\begin{aligned}
\pi[X] & =\pi\left[\sum_{i=1}^{n} m\left(\frac{x_{i}-x_{i-1}}{m} B_{p_{i}+\cdots+p_{n}}^{c}\right)\right] \\
& =f\left(\sum_{i=1}^{n} m f^{-1}\left(v\left(\frac{x_{i}-x_{i-1}}{m}, 1-F\left(x_{i-1}\right)\right)\right)\right) .
\end{aligned}
$$

By the assumption on the function $v(\cdot, \cdot)$, one easily sees that

$$
\lim _{x \searrow 0} \frac{f^{-1}(v(x, q))}{x}=\lim _{v \searrow 0} \frac{f^{-1}(v)}{v} \cdot \lim _{x \searrow 0} \frac{v(x, q)}{x}=\frac{w(q)}{f^{\prime}(0)}=\frac{w(q)}{w(1)} .
$$


Therefore, by the continuity of the function $f(\cdot)$ and $(2.10)$ we derive

$$
\begin{aligned}
\pi[X] & =\lim _{m \rightarrow+\infty} f\left(\sum_{i=1}^{n} m f^{-1}\left(v\left(\frac{x_{i}-x_{i-1}}{m}, 1-F\left(x_{i-1}\right)\right)\right)\right) \\
& =f\left(\sum_{i=1}^{n}\left(x_{i}-x_{i-1}\right) \lim _{m \rightarrow+\infty} \frac{f^{-1}\left(v\left(\left(x_{i}-x_{i-1}\right) / m, 1-F\left(x_{i-1}\right)\right)\right)}{\left(x_{i}-x_{i-1}\right) / m}\right) \\
& =f\left(\sum_{i=1}^{n}\left(x_{i}-x_{i-1}\right) \frac{w\left(1-F\left(x_{i-1}\right)\right)}{w(1)}\right) \\
& =f\left(\frac{1}{w(1)} \int_{0}^{+\infty} w\left(1-F_{X}(x)\right) \mathrm{d} x\right) .
\end{aligned}
$$

This indicates that the formula (2.3) holds for the case where $X$ have only finite supporting points.

2.2. Now we consider the general case, i.e. the risk variable $X$ has a distribution function $F_{X}$ which is supported on the half line $[0,+\infty)$. It is standard in measure theory that the distribution function $F_{X}$ can be approximated by a monotonic sequence of discrete distribution functions $F_{X_{n}}$ of the type given in (2.7). Then, respectively by property A3, the result obtained in step 2.1 , and the continuity of the function $f(\cdot)$, we have

$$
\begin{aligned}
\pi[X] & =\lim _{n \rightarrow+\infty} \pi\left[X_{n}\right] \\
& =\lim _{n \rightarrow+\infty} f\left(\frac{1}{w(1)} \int_{0}^{+\infty} w\left(1-F_{X_{n}}(x)\right) \mathrm{d} x\right) \\
& =f\left(\frac{1}{w(1)} \lim _{n \rightarrow+\infty} \int_{0}^{+\infty} w\left(1-F_{X_{n}}(x)\right) \mathrm{d} x\right) \\
& =f\left(\frac{1}{w(1)} \int_{0}^{+\infty} w\left(1-F_{X}(x)\right) \mathrm{d} x\right),
\end{aligned}
$$

where, at the last step we applied the monotone convergence theorem. Thus, we obtain the announced result (2.3) for the general case.

This ends the proof of Theorem 2.1.

\section{The Haezendonck risk measure}

Let $X$ be a random variable with range $-\infty \leq \min [X] \leq \max [X] \leq+\infty$. In this section we aim at a new risk measure. For this purpose we introduce a non-negative, strictly increasing, and continuous function $\phi(\cdot)$ with $\phi(0)=0, \phi(1)=1$, and $\phi(+\infty)=+\infty$. Then, for any $x \in(-\infty,+\infty)$ and any $\pi>x$, applying the devices developed in Goovaerts et al. $\left(2003_{b}\right)$ we obtain that

$$
\operatorname{Pr}[X>\pi]=\operatorname{Pr}[X-x>\pi-x] \leq \mathrm{E}\left[\phi\left(\frac{(X-x)_{+}}{\pi-x}\right)\right] .
$$


For the inequality (3.1) to make sense, the bivariate function $\phi(\cdot)$ and the r.v. $X$ have to satisfy

$$
\mathrm{E}[\phi(X)]<+\infty
$$

Hence, by assuming (3.2) the random variables considered are restricted to the family

$$
\mathbb{X}_{\phi}=\{X: \mathrm{E}[\phi(X)]<+\infty\}
$$

This will be assumed automatically in this section. For any given value $0<\alpha<1$, observe the equation

$$
\mathrm{E}\left[\phi\left(\frac{(X-x)_{+}}{\pi-x}\right)\right]=1-\alpha
$$

It has no solution in case $x \geq \max [X]$; in this case we assume by convention that the solution is $+\infty$. So we only consider the case $-\infty<x<\max [X]$. Recall the restrictions made on the function $\phi(\cdot)$. By the monotone convergence theorem we easily see that

$$
\lim _{\pi \searrow x} \mathrm{E}\left[\phi\left(\frac{(X-x)_{+}}{\pi-x}\right)\right]=\phi(+\infty) \operatorname{Pr}[X>x]=+\infty
$$

and that

$$
\lim _{\pi \nearrow+\infty} \mathrm{E}\left[\phi\left(\frac{(X-x)_{+}}{\pi-x}\right)\right]=\phi(0) \operatorname{Pr}[X>x]=0 .
$$

Hence for any $-\infty<x<\max [X]$ and $0<\alpha<1$, the equation (3.4) has a unique solution, say $\pi_{\alpha}[X, x]$, which lies in the interval $(x,+\infty)$. By $(3.1)$, we also get to know that the solution $\pi_{\alpha}[X, x]$ gives an upper bound for the quantile $F_{X}^{-1}(\alpha)$.

We summarize this into a theorem as follows:

Theorem 3.1. Let $X$ be a risk variable, and let $\phi(\cdot)$ be a non-negative, strictly increasing, and continuous function with $\phi(0)=0, \phi(1)=1$, and $\phi(+\infty)=+\infty$. Then for any $-\infty<x<\max [X]$ and $0<\alpha<1$, the equation (3.4) has a unique solution $\pi_{\alpha}[X, x]$ satisfying the inequality

$$
\max \left\{x, F_{X}^{-1}(\alpha)\right\} \leq \pi_{\alpha}[X, x]
$$

For the readers who want some mathematical sophistication, Theorem 3.1 can be expressed in terms of Young functions and Orlicz norms. That is the reason why we called it Orlicz risk measure in Haezendonck and Goovaerts (1982). The origin of the principle stems from an idea that originated from the Swiss premium calculation principle that is due to Bühlmann et al. (1977).

Now we introduce an important notion in this section: 
Definition 3.1. Let $X$ be a risk variable, let $\phi(\cdot)$ be a non-negative, strictly increasing, and continuous function with $\phi(0)=0, \phi(1)=1$, and $\phi(+\infty)=+\infty$, and let $0<\alpha<1$ be arbitrarily fixed. We consider

$$
\pi_{\alpha}[X]=\inf _{-\infty<x<\max [X]} \pi_{\alpha}[X, x]
$$

as the risk measure for the risk variable $X$, where $\pi_{\alpha}[X, x]$ is the unique solution of the equation (3.4). In honor of the late J. Haezendonck we call it the Haezendonck risk measure, which is a minimal Orlicz risk measure.

Recall our convention that $\pi_{\alpha}[X, x]=\infty$ for $x \geq \max [X]$. The definition (3.6) can also be rewritten as

$$
\pi_{\alpha}[X]=\inf _{-\infty<x<\infty} \pi_{\alpha}[X, x]
$$

Theorem 3.2. Let $X$ be a risk variable, and let $\phi(\cdot)$ be a non-negative, strictly increasing, and continuous function with $\phi(0)=0, \phi(1)=1$, and $\phi(+\infty)=+\infty$. Then the Haezendonck risk measure $\pi_{\alpha}[X]$ satisfies

$$
F_{X}^{-1}(\alpha) \leq \pi_{\alpha}[X] \leq \max [X]
$$

Proof. The left-hand side of (3.7) is a direct consequence of Theorem 3.1. To prove the right-hand side of (3.7), we choose $x=F_{X}^{-1}\left(\alpha^{\prime}\right)$ for some $\alpha<\alpha^{\prime}<1$ and observe that

$$
\mathrm{E}\left[\phi\left(\frac{(X-x)_{+}}{\max [X]-x}\right)\right] \leq \operatorname{Pr}[X>x] \leq 1-\alpha^{\prime}<1-\alpha
$$

Comparing this with the equation (3.4) leads to $\pi_{\alpha}[X, x] \leq \max [X]$. Hence $\pi_{\alpha}[X] \leq$ $\max [X]$. This ends the proof of Theorem 3.2 .

Example 3.1. Now we specify the risk variable in Definition 3.1 as $B_{q}$, a Bernoulli variable with

$$
\operatorname{Pr}\left[B_{q}=1\right]=1-\operatorname{Pr}\left[B_{q}=0\right]=q \in[0,1] .
$$

Let $\phi(x)=x$ for $x \geq 0$, and let $-\infty<x<1$ and $0<\alpha<1$ be arbitrarily given. In case $-\infty<x<0$, the equation (3.4) is specified as

$$
(1-q) \frac{-x}{\pi-x}+q \frac{1-x}{\pi-x}=1-\alpha,
$$

whereas in case $0 \leq x<1$, it is specified as

$$
q \frac{1-x}{\pi-x}=1-\alpha
$$


Clearly, the equation (3.8) has a unique solution that

$$
\pi_{\alpha}\left[B_{q}, x\right]=\frac{q-x}{1-\alpha}, \quad-\infty<x<0,
$$

whereas the equation (3.9) has a unique solution that

$$
\pi_{\alpha}\left[B_{q}, x\right]=\frac{q}{1-\alpha}+\frac{1-\alpha-q}{1-\alpha}, \quad 0 \leq x<1 .
$$

Simple analysis gives that

$$
\pi_{\alpha}\left[B_{q}\right]=\inf _{-\infty<x<\max [X]} \pi_{\alpha}\left[B_{q}, x\right]=\min \left\{\frac{q}{1-\alpha}, 1\right\} .
$$

Theorem 3.3. Let $\pi_{\alpha}[X]$ be the Haezendonck risk measure of a risk variable $X$ with $\phi(\cdot)$ satisfying the restrictions in Definition 3.1, and let $0<\alpha<1$ be arbitrarily given. Then we have

B1. Monotonicity: If $X \leq_{1} Y$ then $\pi_{\alpha}[X] \leq \pi_{\alpha}[Y]$;

B2. Positive Homogeneity: $\pi_{\alpha}[c X]=c \pi_{\alpha}[X]$ for any $c>0$;

B3. Subadditivity: If $\phi(\cdot)$ is convex, then $\pi_{\alpha}[X+Y] \leq \pi_{\alpha}[X]+\pi_{\alpha}[Y]$;

B4. Translation Invariance: $\pi_{\alpha}[X+a]=\pi_{\alpha}[X]+$ a for any $a$;

B5. Preservation of convex ordering: If $\phi(\cdot)$ is convex, then $X \leq_{c x} Y \Longrightarrow \pi_{\alpha}(X) \leq \pi_{\alpha}(Y)$.

Proof. B1. Obviously, $X \leq_{1} Y$ indicates that $\max [X] \leq \max [Y]$. On the other hand, by the equation (3.4) once again we easily see that the inequality

$$
\pi_{\alpha}[X, y] \leq \pi_{\alpha}[Y, y]
$$

holds for any $-\infty<y<\max [X] \leq \max [Y]$. Hence we have

$$
\begin{aligned}
\pi_{\alpha}[Y] & =\min \left\{\inf _{-\infty<y<\max [X]} \pi_{\alpha}[Y, y], \inf _{\max [X] \leq y<\max [Y]} \pi_{\alpha}[Y, y]\right\} \\
& \geq \min \left\{\inf _{-\infty<x<\max [X]} \pi_{\alpha}[X, y], \max [X]\right\} \\
& =\min \left\{\pi_{\alpha}[X], \max [X]\right\} \\
& =\pi_{\alpha}[X] .
\end{aligned}
$$

This proves the property of the monotonicity. 
B2. For any $c>0$ and any $-\infty<x<\max [c X]$, by the equation (3.4) we easily see that

$$
\pi_{\alpha}[c X, x]=c \pi_{\alpha}[X, x / c]
$$

Hence

$$
\begin{aligned}
\pi_{\alpha}[c X] & =\inf _{-\infty<x<\max [c X]} \pi_{\alpha}[c X, x] \\
& =c \inf _{-\infty<x<\max [c X]} \pi_{\alpha}[X, x / c] \\
& =c \inf _{-\infty<y<\max [X]} \pi_{\alpha}[X, y] \\
& =c \pi_{\alpha}[X] .
\end{aligned}
$$

This proves the property of the positive homogeneity.

B3. For any two random variables $X$ and $Y$, it is obvious that

$$
\max [X+Y] \leq \max [X]+\max [Y]
$$

For any $-\infty<x<\max [X]$ and $-\infty<y<\max [Y]$, we derive

$$
\begin{aligned}
& \mathrm{E}\left[\phi\left(\frac{(X+Y-x-y)_{+}}{\pi_{\alpha}[X, x]+\pi_{\alpha}[Y, y]-x-y}\right)\right] \\
\leq & \mathrm{E}\left[\phi\left(\frac{(X-x)_{+}+(Y-y)_{+}}{\left(\pi_{\alpha}[X, x]-x\right)+\left(\pi_{\alpha}[Y, y]-y\right)}\right)\right] \\
\leq & \frac{\pi_{\alpha}[X, x]-x}{\left(\pi_{\alpha}[X, x]-x\right)+\left(\pi_{\alpha}[Y, y]-y\right)} \mathrm{E}\left[\phi\left(\frac{(X-x)_{+}}{\pi_{\alpha}[X, x]-x}\right)\right] \\
= & 1-\alpha, \quad+\frac{\pi_{\alpha}[Y, y]-y}{\left(\pi_{\alpha}[X, x]-x\right)+\left(\pi_{\alpha}[Y, y]-y\right)} \mathrm{E}\left[\phi\left(\frac{(Y-y)_{+}}{\pi_{\alpha}[Y, y]-y}\right)\right]
\end{aligned}
$$

where we have used the convexity of the function $\phi$ and the inequality (3.5). This proves that, for any $-\infty<x<\max [X]$ and $-\infty<y<\max [Y]$,

$$
\pi_{\alpha}[X+Y, x+y] \leq \pi_{\alpha}[X, x]+\pi_{\alpha}[Y, y] .
$$

Recall our convention that $\pi_{\alpha}[X, x]=+\infty$ for $x \geq \max [X]$. Hence the inequality (3.11) holds for any $-\infty<x<+\infty$ and $-\infty<y<+\infty$. Therefore by (3.10) and (3.11) we have

$$
\begin{aligned}
\pi_{\alpha}[X+Y] & =\inf _{-\infty<x+y<\max [X+Y]} \pi_{\alpha}[X+Y, x+y] \\
& =\inf _{-\infty<x+y<\max [X]+\max [Y]} \pi_{\alpha}[X+Y, x+y] \\
& \leq \inf _{-\infty<x+y<\max [X]+\max [Y]}\left(\pi_{\alpha}[X, x]+\pi_{\alpha}[Y, y]\right) \\
& =\underset{-\infty<x<\max [X],-\infty<y<\max [Y]}{ }\left(\pi_{\alpha}[X, x]+\pi_{\alpha}[Y, y]\right) \\
& =\inf _{-\infty<x<\max [X]} \pi_{\alpha}[X, x]+\inf _{-\infty<y<\max [Y]} \pi_{\alpha}[Y, y] \\
& =\pi_{\alpha}[X]+\pi_{\alpha}[Y] .
\end{aligned}
$$


where the facts

$$
\inf _{-\infty<x+y<\max [X+Y]} \pi_{\alpha}[X+Y, x+y]=\inf _{-\infty<x+y<\max [X]+\max [Y]} \pi_{\alpha}[X+Y, x+y]
$$

and

$$
\inf _{-\infty<x+y<\max [X]+\max [Y]}\left(\pi_{\alpha}[X, x]+\pi_{\alpha}[Y, y]\right)=\inf _{-\infty<x<\max [X],-\infty<y<\max [Y]}\left(\pi_{\alpha}[X, x]+\pi_{\alpha}[Y, y]\right)
$$

are natural consequences of our convention. Hence the property of the Subadditivity holds.

B4. Analogously, for any $-\infty<a<+\infty$ and any $-\infty<x<\max [X+a]$, by the equation (3.4) we easily see that $\pi_{\alpha}[X+a, x]=\pi_{\alpha}[X, x-a]+a$. It follows that

$$
\begin{aligned}
\pi_{\alpha}[X+a] & =\inf _{-\infty<x<\max [X+a]} \pi_{\alpha}[X+a, x] \\
& =\inf _{-\infty<x<\max [X+a]} \pi_{\alpha}[X, x-a]+a \\
& =\inf _{-\infty<y<\max [X]} \pi_{\alpha}[X, y]+a \\
& =\pi_{\alpha}[X]+a .
\end{aligned}
$$

This proves the property of the translation invariance.

B5. We know that $X \leq_{c x} Y$ means that $\mathrm{E} \varphi(X) \leq \mathrm{E} \varphi(Y)$ holds for all convex function $\varphi(\cdot)$ whenever the expectations involved exist. Now we specify the function $\varphi(\cdot)$ as

$$
\varphi(\pi)=\varphi_{x}(\pi)=\phi\left(\frac{(X-x)_{+}}{\pi-x}\right) \quad \text { for } \pi>x .
$$

Since the function $\phi(\cdot)$ is assumed to be convex, the function $\varphi(\pi)$ is also convex in $\pi \in$ $(x,+\infty)$. It follows that

$$
\mathrm{E} \phi\left(\frac{(X-x)_{+}}{\pi-x}\right) \leq \mathrm{E} \phi\left(\frac{(Y-x)_{+}}{\pi-x}\right) .
$$

This indicates that $\pi_{\alpha}[X, x] \leq \pi_{\alpha}[Y, x]$ for all $x \in(-\infty,+\infty)$. Hence $\pi_{\alpha}(X) \leq \pi_{\alpha}(Y)$ holds.

This ends the proof of Theorem 3.3.

The Orlicz and Haezendonck insurance premium principles and/or risk measures have some interesting ordering consequences. Now we consider the following definitions.

Definition 3.2. Let $\phi_{1}(\cdot)$ and $\phi_{2}(\cdot)$ be two real functions on $(0,+\infty)$. We say $\phi_{2}(\cdot)$ is convex (concave) in $\phi_{1}(\cdot)$ if and only if $\phi_{2} \phi_{1}^{-1}(\cdot)$ is convex (concave).

We have the following result: 
Theorem 3.4. Let $\phi_{i}(\cdot), i=1,2$, be two continuous and strictly increasing functions with $\phi_{i}(x)=x$ for $x \in[0,1]$ and $\phi_{i}(+\infty)=+\infty$, let $\pi_{\alpha}^{(i)}[X, x], i=1,2$, be the solutions of (3.4) with $\phi_{i}(\cdot)$, and let the corresponding Haezendonck risk measures be

$$
\pi_{\alpha}^{(i)}[X]=\inf _{-\infty<x<\max [X]} \pi_{\alpha}^{(i)}[X, x], \quad i=1,2 .
$$

1). If $\phi_{2}(\cdot)$ is convex in $\phi_{1}(\cdot)$ then $\pi_{\alpha}^{(1)}[X, x] \leq \pi_{\alpha}^{(2)}[X, x]$, hence $\pi_{\alpha}^{(1)}[X] \leq \pi_{\alpha}^{(2)}[X]$;

2). If $\phi_{2}(\cdot)$ is concave in $\phi_{1}(\cdot)$ then $\pi_{\alpha}^{(1)}[X, x] \geq \pi_{\alpha}^{(2)}[X, x]$, hence $\pi_{\alpha}^{(1)}[X] \geq \pi_{\alpha}^{(2)}[X]$.

Proof. We only give the proof of the first result since the proof for the second one can be given similarly. By the definition of $\pi_{\alpha}^{(2)}[X, x]$, we have

$$
\mathrm{E}\left[\phi_{2}\left(\frac{(X-x)_{+}}{\pi_{\alpha}^{(2)}[X, x]-x}\right)\right]=1-\alpha,
$$

Since the compound function $\phi_{2} \phi_{1}^{-1}(\cdot)$ is convex on $(0,+\infty)$, by Jensen's inequality we obtain

$$
\begin{aligned}
\mathrm{E}\left[\phi_{2}\left(\frac{(X-x)_{+}}{\pi_{\alpha}^{(1)}[X, x]-x}\right)\right] & =\mathrm{E}\left[\phi_{2} \phi_{1}^{-1} \phi_{1}\left(\frac{(X-x)_{+}}{\pi_{\alpha}^{(1)}[X, x]-x}\right)\right] \\
& \geq \phi_{2} \phi_{1}^{-1}\left(\mathrm{E}\left[\phi_{1}\left(\frac{(X-x)_{+}}{\pi_{\alpha}^{(1)}[X, x]-x}\right)\right]\right) \\
& =\phi_{2} \phi_{1}^{-1}(1-\alpha) \\
& =1-\alpha .
\end{aligned}
$$

Comparing (3.13) with (3.12) yields that $\pi_{\alpha}^{(2)}[X, x] \geq \pi_{\alpha}^{(1)}[X, x]$. This ends the proof of Theorem 3.4.

The following is an immediate consequence of Theorem 3.4:

Corollary 3.1. The Tail-VaR, which is defined by

$$
\operatorname{TVaR}_{\alpha}[X]=F_{X}^{-1}(\alpha)+\frac{1}{1-\alpha} \mathrm{E}\left[\left(X-F_{X}^{-1}(\alpha)\right)_{+}\right], \quad \alpha \in(0,1),
$$

is the smallest one among those Haezendonck risk measures $\pi_{\alpha}[X]$ that correspond to strictly increasing and convex functions $\phi(\cdot)$ satisfying $\phi(x)=x$ for $0<x<1$, and is the largest one among those Haezendonck risk measures $\pi_{\alpha}[X]$ that correspond to strictly increasing and concave functions $\phi(\cdot)$ satisfying $\phi(x)=x$ for $0<x<1$ and $\phi(+\infty)=+\infty$.

Proof. Let the notation in Theorem 3.4 be valid. If we choose $\phi_{1}(x)=x$ for $x \in(0, \infty)$, then by the equation (3.4) we derive that

$$
\pi_{\alpha}^{(1)}[X, x]=x+\frac{1}{1-\alpha} \mathrm{E}\left[(X-x)_{+}\right] \quad \text { for all }-\infty<x<\max [X]
$$


Taking the inf over the range $x \in(-\infty, \max [X])$ yields that $\pi_{\alpha}^{(1)}[X]=T \operatorname{Va} R_{\alpha}[X]$. Hence the corollary can be proved by Theorem 3.4.

Corollary 3.1 indicates that the Tail-VaR characterizes simply the intermediate case among the Haezendonck risk measures based on convex (concave) functions $\phi(\cdot)$ on $(0,+\infty)$.

\section{References}

[1] Bühlmann, H., 1970. Mathematical Methods in Risk Theory. Springer-Verlag, Berlin.

[2] Bühlmann, H., Gagliardi, B., Gerber, H.U., Straub, E., 1977. Some inequalities for stop-loss premiums. Astin Bull. 9, no. 1, 75-83.

[3] Gerber, H.U., 1979. An Introduction to Mathematical Risk Theory. Huebner Foundation Monograph 8, distributed by Richard D. Irwin, Inc., Homewood, Illinois.

[4] Gerber, H.U., 1985. On additive principles of zero utility. Insurance Math. Econom. 4, no. 4, 249-251.

[5] Goovaerts, M. J., De Vijlder, F., Haezendonck, J., 1984. Insurance Premiums. NorthHolland Publishing Co., Amsterdam.

[6] Goovaerts, M., Kaas, R., Dhaene, J., 2003a. Economic capital allocation derived from risk measures, North American Actuarial Journal, 7, no. 2.

[7] Goovaerts, M., Kaas, R., Dhaene, J., Tang, Q.H., 2003. A unified approach to generate risk measures. Submitted.

[8] Haezendonck, J., Goovaerts, M., 1982. A new premium calculation principle based on Orlicz norms. Insurance Math. Econom. 1 no. 1, 41-53.

[9] Kaas, R., Goovaerts, M. J., Dhaene, J., Denuit, M., 2001. Modern Actuarial Risk Theory. Dordrecht: Kluwer Acad. Publ.

[10] Wang, S., 1996. Premium calculation by transforming the layer premium density. ASTIN Bulletin, 26, no. 1, 71-92.

[11] Wang, S., Young, V.R., 1998. Ordering risks: expected utility theory versus Yaari's dual theory of risk. Insurance Math. Econom. 22, no. 2, 145-161.

[12] Yaari, M.E., 1987. The dual theory of choice under risk. Econometrica, 55, no. 1, 95115. 


\section{Marc Goovaerts}

Center for Risk and Insurance Studies (CRIS)

Katholieke Universiteit Leuven

B - 3000 Leuven, BELGIUM

E-mail: Marc.Goovaerts@econ.kuleuven.ac.be

ROB KAAS

Department of Quantitative Economics

University of Amsterdam

Roetersstraat 11, 1018 WB Amsterdam, THE NETHERLANDS

E-mail: r.kaas@uva.nl

JAN DhaEne

Center for Risk and Insurance Studies (CRIS)

Katholieke Universiteit Leuven

B - 3000 Leuven, BELGIUM

E-mail: Jan.Dhaene@econ.kuleuven.ac.be

QIHE TANG

Department of Quantitative Economics

University of Amsterdam

Roetersstraat 11, 1018 WB Amsterdam, THE NETHERLANDS

E-mail: q.tang@uva.nl 